\title{
Kommunikative
}

\section{Rahmenbedingungen beim Change Management in der Schule}

\author{
Annika Teerling', Steffen Zitzmann², Jennifer Igler ${ }^{3}$, Theresa Schlitter ${ }^{3}$, Annika Ohle-Peters ${ }^{3}$, \\ Nele McElvany ${ }^{3}$ und Olaf Köller ${ }^{1}$
}

${ }^{1}$ Leibniz-Institut für die Pädagogik der Naturwissenschaften und Mathematik (IPN) an der Universität Kiel

${ }^{2}$ Christian-Albrechts-Universität zu Kiel

${ }^{3}$ Technische Universität Dortmund

\begin{abstract}
Zusammenfassung: Unternehmen und Organisationen stehen unter ständigem Veränderungsdruck. Vor allem seit PISA 2000 können sich auch Schulen in Deutschland diesem nicht mehr entziehen. Der vorliegende Beitrag geht der Frage nach, inwieweit kommunikative Rahmenbedingungen im Veränderungsprozess als relevant für die wahrgenommene Entwicklung der Betroffenen und die Diffusion der Veränderung sowie Schüleroutcomes erscheinen. Mithilfe von Mehrebenenanalysen wird untersucht, inwieweit sich die individuelle Ebene der Betroffenen und die Organisationsebene dabei unterscheiden. Auf Basis einer Stichprobe von $N=66$ Lehrkräften und Schulleitungen kann dabei gezeigt werden, dass auf individueller Ebene vor allem die Eigenschaften der Betroffenen sowie die Häufigkeit der Kooperation und auf Organisationsebene die Zusammensetzung der Projektgruppe als relevant für den Veränderungsprozess erscheinen. Zudem werden Zusammenhänge der Selbstberichte der Lehrkräfte mit Leistungen und motivationalen Orientierungen auf Seiten der Schülerinnen und Schüler $(N=539)$ berichtet.
\end{abstract}

Schlüsselwörter: Change Management, Kommunikation, Organisationsentwicklung, Schulen, Mehrebenenanalysen

Communicative Conditions in Change Management Within Schools

Abstract: Companies and organizations are constantly under pressure to change. And especially after PISA 2000, even schools can no longer avoid this. The present article examines to what extent communicative framework conditions in the change process are relevant for the participants' perception of the development and the diffusion of the change, as well as for student outcomes. Multilevel analyses were performed to explore the extent to which the individual level of those affected and the organizational level differ. Based on a sample of 66 teachers and school principals, it is shown that, on the individual level, the characteristics of the participants as well as the frequency of cooperation seem to be especially relevant for the change process; on the organizational level, the composition of the project group seems to be relevant. Furthermore, the association between the teachers' self-reports and the students' performance and motivational orientation ( $N$ = 539) are reported.

Keywords: change management, communication, organizational development, schools, multilevel analysis

Im Zuge der Globalisierung und der damit einhergehenden internationalen Konkurrenz sowie durch den Fortschritt sind nachhaltige Anpassungseffekte in Unternehmen und Organisationen erforderlich (von Rosenstiel \& Nerdinger, 2011). Was für die Wirtschaft gilt, trifft seit Initiierung der internationalen Schulleistungsstudie PISA, die im Auftrag der Organisation für wirtschaftliche $\mathrm{Zu}$ sammenarbeit und Entwicklung (OECD) die Kompetenzen von 15-jährigen Jugendlichen beim Lesen, in der Mathematik und den Naturwissenschaften erfasst, zum Teil auch auf den Sektor Schule zu. Mit PISA hat ein internationaler Wettbewerb um erfolgreiche Schulsysteme ein- gesetzt. Bei der ersten Studie im Jahr 2000 schnitten deutsche Schülerinnen und Schüler unterdurchschnittlich ab (vgl. Baumert et al., 2001), was zu mehreren Bildungsund Schulreformen führte, als deren Folge die einheitlichen Bildungsstandards in den Bundesländern sowie zahlreiche Programme zur Verbesserung der Kompetenzen der Schülerinnen und Schüler eingeführt wurden und immer noch werden.

Schulen als Organisationen sind somit von verschiedenen Veränderungsprozessen betroffen, wobei im Rahmen von Programmen wie „Bildung durch Sprache und Schrift“ (BiSS) oder „Steigerung der Effizienz des ma- 


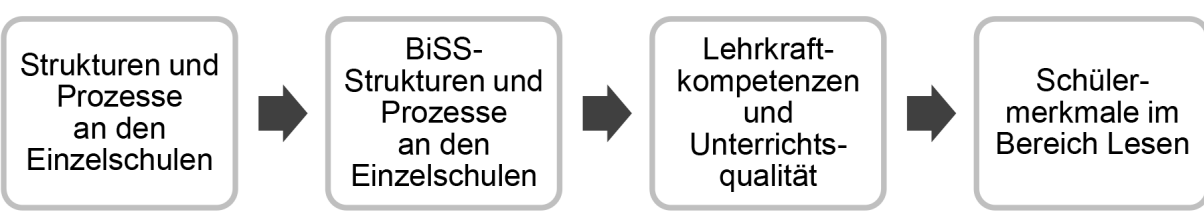

Abbildung 1. Theoretisches Wirkmodell als Grundlage von BiSS-EvalLesen (McElvany et al., 2018). thematisch-naturwissenschaftlichen Unterrichts" (SINUS) die Lehrkräfte fortgebildet sowie mit entsprechenden Unterrichtsmaterialien ausgestattet werden. Dadurch, dass zudem entsprechende Strukturen und Prozesse in den Einzelschulen - wie zum Beispiel Projekt- oder Steuergruppen - geschaffen werden, sollen die Art und Weise des Unterrichtens verbessert und schlussendlich die sprachlichen oder mathematisch-naturwissenschaftlichen Kompetenzen der Schülerinnen und Schüler gefördert werden (vgl. Abbildung 1). Schlussendlich sollen solche Programme damit zu einem besseren Abschneiden in Schulleistungsstudien beitragen. Solche Implementationsprozesse sind jedoch vor allem im Kontext von Schule mit Schwierigkeiten verbunden, da Lehrkräfte viel Autonomie beim Unterrichten besitzen (Altrichter \& Eder, 2004). Insbesondere vor diesem Hintergrund stellt die Kooperation zwischen den Lehrkräften ein zentrales Element, aber auch eine große Herausforderung in entsprechenden Implementationsprozessen dar (Steinert, Klieme, Maag Merki, Döbrich, Halbheer \& Kunz, 2006; Jäger, 2004; Gräsel, Jäger \& Willke, 2006, Bergmann \& Rollett, 2008; Goldenbaum, 2013), welche in der vorliegenden Arbeit - unterschieden im Hinblick auf die Dimensionen Häufigkeit und Qualität - als bedeutsamer Faktor betrachtet werden soll. Eng damit verbunden sind auf individueller Ebene die (kommunikativen) Eigenschaften der beteiligten Personen (Rogers, 2003; Hall \& Hord, 2006; Batinic, Haupt \& Wieselhuber, 2006) und auf organisationaler Ebene die Zusammensetzung der Projektgruppe (Argote, McEvily \& Reagans, 2003; Edmondson, Bohmer \& Pisano, 2001; Zacher et al., 2008).

Der vorliegenden Studie liegt das theoretische Wirkmodell (Abbildung 1) im Rahmen des BiSS-Programms zugrunde, wobei sich hier auf die Evaluation des Bereichs Lesen an Grundschulen (BiSS-EvalLesen) fokussiert wird. Im Hinblick auf die Einzelschulen werden dabei kommunikative und kooperative Strukturen und Prozesse im Rahmen des BiSS-Programms in den Blick genommen und als Prädiktoren für die Art und Weise der Unterrichtsentwicklung seit Programmbeginn überprüft, wobei letzteres über die wahrgenommene Entwicklung der Lehrkräfte abgebildet wird. Um darüber hinaus eine Veränderung auf Schulebene zu erfassen, werden zudem $\mathrm{Zu}$ sammenhänge mit der Diffusion der Veränderung (Schulleitungsvariable) als organisationalen Indikator für veränderte Lehrkraftkompetenzen und Unterrichtsqualität innerhalb der Schulen überprüft. Dem theoretischen Wirkmodell weiter folgend wurde darüber hinaus untersucht, inwieweit die wahrgenommene Entwicklung und die Diffusion der Veränderung prädiktiv für die Schülerkompetenzen im Bereich Lesen sind.

Im vorliegenden Beitrag wurde dabei ein Mehrebenendesign gewählt, um die Mikro- und die Makroperspektiven miteinander zu kombinieren. Es werden also individuelle Faktoren der Beteiligten mit organisationalen Kontextfaktoren verbunden. Durch die Befragung der Lehrkräfte liegen Daten vor, die auf individueller Ebene und auf Aggregatebene betrachtet werden. Durch die Befragung der Schulleitungen liegen zudem Vorgesetztenurteile vor. Laut van Dick und Kollegen (2005) ist es somit sinnvoll, die Effekte der Variablen, die sowohl auf individueller Ebene als auch auf Schulebene angesiedelt sind, zu betrachten. Die Mehrebenenanalysen ermöglichen damit einen optimalen Erkenntnisfortschritt (van Dick, 2005), indem neben den Effekten auf individueller Ebene (hier die Lehrkräfte) auch mögliche Kontexteffekte (hier die Schulebene) geprüft werden (Langer, 2009). So werden nicht nur individuelle Wahrnehmungen berücksichtigt, sondern auch die Schulkultur bzw. das Arbeitsklima an der Schule.

Auf individueller Ebene werden als Strukturen und Prozesse die (kommunikativen) Eigenschaften der Beteiligten sowie die Qualität und Häufigkeit der Kooperation überprüft. Auf Organisationsebene werden diese Skalen als manifeste Schulmittelwerte ebenfalls berücksichtigt, um die Schulkultur bzw. das Arbeitsklima abzubilden. Darüber hinaus wird hier zudem die Zusammensetzung der Projektgruppe (Schulleitungsangabe) hinzugezogen.

Anhand der Ergebnisse sollen die individuellen und organisationalen Prozesse und Strukturen an den Einzelschulen besser verstanden werden. Ziel ist es zudem, konkrete Hinweise $\mathrm{zu}$ formulieren, sodass bestehende und künftige Programme eine angestrebte Veränderung auf Seiten der Lehrkräfte sowie schlussendlich eine tatsächliche Verbesserung der Kompetenzen auf Seiten der Schülerinnen und Schüler fördern können. 


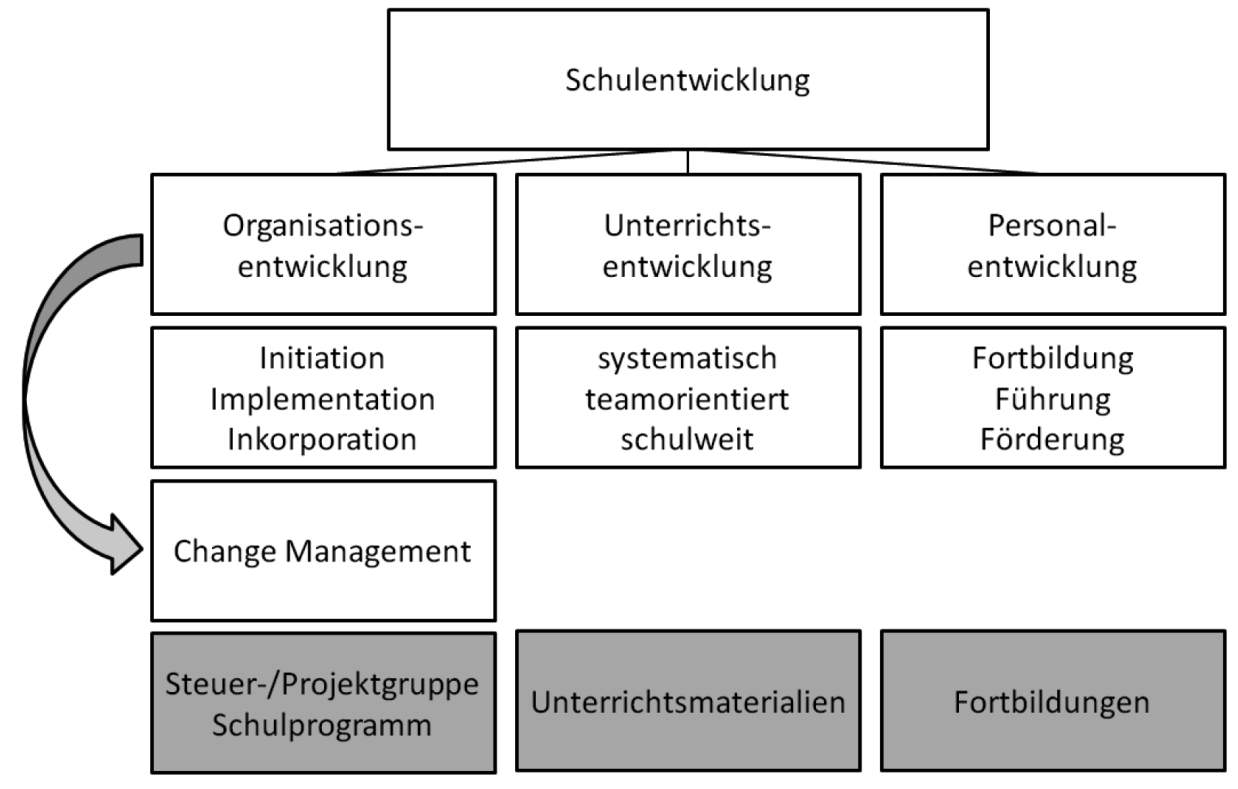

Abbildung 2. Modell der Schulentwicklung nach Rolff (2007, 2012a); eingegraut: Bestandteil von BiSS.

\section{Schulentwicklung: Organisations-, Unterrichts- und Personalentwicklung sowie Change Management}

Eine Form des geplanten Wandels, die in den letzten Jahren zunehmend an Bedeutung gewonnen hat, ist das Change Management, das in der Arbeits- und Organisationspsychologie alle Ansätze eines gezielt gesteuerten, umfassenden organisationalen Wandels umfasst (Nerdinger, 2019). Mit Formen wie dem Business Reengineering, Lean Management oder Total Quality Management sollen dabei die Kosten gesenkt, die Effizienz gesteigert, die Kundenorientierung verbessert oder eine umfassende Qualitätssicherung im Unternehmen erreicht werden. Dabei unterscheidet sich das Change Management von der Organisationsentwicklung laut Nerdinger (2019) vor allem darin, dass diese Ansätze ohne Einbezug der Betroffenen von Beratern entworfen und anschließend umgesetzt werden. Organisationsentwicklung bezeichnet hingegen ebenfalls eine Form des geplanten Wandels von Organisationen, hier sind die Betroffenen jedoch am Prozess beteiligt. Der Wandel wird dabei durch erfahrungsgeleitete Lern- und Problemlöseprozesse herbeigeführt, die weniger die Produktivität, sondern die Lebensqualität und die Problemlösefähigkeit in der Organisation verbessern sollen (Nerdinger, 2019).

Change Management und Organisationsentwicklung finden sich im Hinblick auf die Organisation Schule im Kontext der Forschung zur Schulentwicklung (Rolff, 2007; Rolff, 2012a). Hierbei stellen Organisations-, Unterrichts- und Personalentwicklung Komponenten der Schulentwicklung dar, die als Synthese wirken. Organisa- tionsentwicklung bezeichnet dabei die Weiterentwicklung der Organisation von innen heraus und durch deren Mitglieder selbst, womit der Begriff hier ähnlich wie in der Arbeits- und Organisationspsychologie verstanden wird, da die Betroffenen am Prozess beteiligt sind. Das Change Management stellt eine Weiterentwicklung der Organisationsentwicklung dar, bei der die Rolle der Führung sowie Evaluation und Qualitätsmanagement stärker in den Blick genommen werden (Rolff, 2012a). Ähnlich wie in der Arbeits- und Organisationspsychologie verlagert sich der Fokus somit von der Mitwirkung der Betroffenen auf die Steuerung und Kontrolle von oben.

Programme wie BiSS oder SINUS lassen sich in dem Modell zur Schulentwicklung nach Rolff (2012a) verorten und sprechen dabei alle drei Komponenten an (Abbildung 2). Im Rahmen der Programme werden die Lehrkräfte fortgebildet (Personalentwicklung), es werden Unterrichtsmaterialien und -methoden zur Verfügung gestellt (Unterrichtsentwicklung) und die Beteiligten werden angehalten, Steuer- oder Projektgruppen zu gründen, kooperative Strukturen zu schaffen und die Programme in ihr Schulprogramm aufzunehmen (Organisationsentwicklung) (McElvany, Ohle-Peters, Igler, Schlitter, Teerling, Asseburg \& Köller, 2018; Fischer, Rieck \& Döhring, 2019). Die Maßnahmen, auf die sich BiSS-EvalLesen bezieht, sind dabei zudem fachübergreifend angelegt, da hier eine Leseförderung über die Fächer hinweg angestrebt wird. Entsprechend müssen auch die Lehrkräfte einbezogen werden, die nicht dem Fach Deutsch angehören, weshalb die Unterrichtsentwicklung schulweit angelegt ist. Sowohl BiSS als auch SINUS sind dabei Programme, in denen die Fortbildungen und Materialien 
hierarchisch von oben nach unten angelegt waren (topdown) und bei denen der geplante Wandel wissenschaftlich begleitet und evaluiert wurde. Die Umsetzung der Maßnahmen konnten die Schulen hingegen eigenständig gestalten (bottom-up). Hier waren die Betroffenen angehalten, den Wandel von innen heraus voranzutreiben. Im Rahmen der genannten Programme lassen sich somit sowohl Komponenten aus der Organisationsentwicklung als auch aus dem Change Management finden, wobei die vorliegende Arbeit vor allem am Change Management ansetzt, da dieses durch Führung und Evaluation weitgehend erkenntnisgeleitet gesteuert werden kann.

Kooperative Strukturen und Prozesse in den Einzelschulen zu schaffen stellt aufgrund des „Autonomie-Paritätsmusters" eine Herausforderung bei schulischen Implementationsprozessen dar (Altrichter \& Eder, 2004). Zum einen können Lehrkräfte ihren Beruf - wie bereits genannt - sehr unabhängig und mit viel Spielraum ausüben (Autonomie) und zum anderen besagen die beruflichen Konventionen, dass keine Lehrkraft in die Angelegenheiten einer anderen Lehrkraft eingreift (Parität). Lehrkräfte können ihren Unterricht somit ihr Berufsleben lang ausüben, ohne dass ihre Arbeit - von einigen Unterrichtsbesuchen mal abgesehen - von den Kolleginnen und Kollegen bewertet und eingeschätzt wird (Altrichter \& Eder, 2004). Aber nur durch entsprechende Rückmeldungen können Entwicklungsprozesse reflektiert und vorangetrieben werden. Im Rahmen von Kooperation müssen Lehrkräfte ihren Unterricht jedoch öffnen und offen mit ihrer eigenen Arbeitsweise umgehen. Folglich müssen die individuellen und organisationalen Strukturen und Prozesse an den Einzelschulen besser verstanden werden, die dann zu einem entsprechenden Entwicklungsprozess auf Seiten der Lehrkräfte und schlussendlich der Schülerinnen und Schüler führen können.

Die vorliegende Arbeit soll anhand des theoretischen Wirkmodells auf individueller Ebene sowie auf Organisationsebene untersuchen, inwieweit sich kommunikative Strukturen und Prozesse an den Einzelschulen als relevant im Implementationsprozess identifizieren lassen, und folgende Hypothesen prüfen:

1. Die von den Beteiligten selbst berichteten kommunikativen Strukturen und Prozesse - also die (kommunikativen) Eigenschaften sowie die Qualität und die Häufigkeit der Kooperation - sind auf individueller Ebene positiv prädiktiv für die mentale, sensible und praktische Entwicklung der Betroffenen.

Entsprechend des theoretischen Wirkmodells (Abbildung 1) wird davon ausgegangen, dass die kommuni- kativen Strukturen und Prozesse im Rahmen des Programms auf individueller Ebene die Lehrkraftkompetenzen - hier operationalisiert über die wahrgenommene Entwicklung der Betroffenen seit Programmbeginn im Implementationsprozess bedingen.

2. Die kommunikativen Strukturen und Prozesse sind - im Hinblick auf die (kommunikativen) Eigenschaften, die Qualität und die Häufigkeit der Kooperation in Form von Aggregateinheiten - sowie die Zusammensetzung der Projektgruppe als Schulkultur bzw. Arbeitsklima auf Organisationsebene positiv prädiktiv für (a) die mentale, sensible und praktische Entwicklung der Betroffenen sowie (b) die Diffusion der Veränderung.

Ebenfalls aufgrund des theoretischen Wirkmodells (Abbildung 1) wird erwartet, dass die kommunikativen Strukturen und Prozesse im Rahmen des Programms auf Organisationsebene die Lehrkraftkompetenzen sowie eine Veränderung auf Seiten des Lehrkraftkollegiums - hier operationalisiert über die Diffusion der Veränderung - vorhersagen.

3. Die (a) mentale, sensible und praktische Entwicklung der Betroffenen sowie die (b) Diffusion der Veränderung sind positiv prädiktiv für die Schülerkompetenzen im Bereich Lesen. ${ }^{1}$

Dem theoretischen Wirkmodell (Abbildung 1) weiter folgend wird angenommen, dass die Entwicklung der Betroffenen sowie die Diffusion der Veränderung sich schlussendlich in den Schülermerkmalen im Bereich Lesen - hier operationalisiert über die Schülerkompetenzen - niederschlägt.

\section{Methode}

Die im vorliegenden Beitrag berichteten Befunde stammen aus einer Teiluntersuchung (BiSS-EvalLesen) im Rahmen des BiSS-Programms, das die Implementation von Angeboten zur Sprachdiagnostik und -förderung sowie Lesediagnostik und -förderung wissenschaftlich untersucht (vgl. Becker-Mrotzek, Hasselhorn, Roth \& Stanat, 2016). Das Programm wurde 2012 als Forschungsund Entwicklungsprogramm initiiert, um die über die vergangenen Jahre bundesweit eingeführten Angebote zur Sprachdiagnostik und -förderung sowie zur Lesediagnostik und -förderung vom Elementar- bis Sekundarbereich im Hinblick auf ihre Wirksamkeit und Effizienz sowie die Fortbildung und Weiterqualifizierung der teilnehmenden Fachkräfte wissenschaftlich zu überprüfen und

\footnotetext{
Da es sich hierbei um gerichtete Hypothesen handelt, wird die Signifikanz einseitig getestet. Entsprechend werden die von Mplus ausgegeben Signifikanzen halbiert.
} 
weiterzuentwickeln (vgl. Becker-Mrotzek et al., 2016). Bereits bestehende Befunde im Rahmen des Evaluationsprojektes zeigen dabei, dass in den hier betrachteten Projektschulen vor allem die Schulleitungen, Externe (u.a. Schulämter, Ministerium) sowie einzelne Lehrkräfte und nur selten das Kollegium die Teilnahme am Programm initiierten. Da verantwortliche Personen somit stellvertretend für die Schulgemeinschaft die Bewerbung für die Teilnahme am Programm einreichen konnten, mussten die Kolleginnen und Kollegen in den Schulen vor Ort hierfür zumeist top-down gewonnen werden und konnten entsprechend Vorbehalte und Widerstände gegenüber dem Programm mitbringen (McElvany et al., 2018). Die Implementation der BiSS-Maßnahmen in den Schulen vor Ort erforderte insofern ein Change Management, da die Programmverantwortlichen bestrebt waren, die Lehrkräfte durch Fortbildungen und Materialien aktiv zu beeinflussen, sodass sie die neuen oder weiterentwickelten Methoden sowohl in ihre individuelle Arbeitsroutine dem Planen und Durchführen von Unterricht - als auch in die Organisation generell - zum Beispiel durch die Aufnahme von (regelmäßigen) BiSS-Maßnahmen in das Schulprogramm - integrieren.

\section{Stichprobe}

Innerhalb des BiSS-Programms evaluierte BiSS-EvalLesen von 2015 bis 2018 Konzepte und Maßnahmen der fachübergreifenden Leseförderung und -diagnostik an Grundschulen in sieben Verbünden bzw. Bundesländern (McElvany et al., 2018). Die entsprechenden Schulen bearbeiteten dabei die Module 3 (Diagnose und Förderung der Leseflüssigkeit und ihrer Voraussetzungen) und 4 (Diagnose und Förderung des Leseverständnisses). Die hier verwendeten Fragebogendaten wurden im Rahmen des zweiten Messzeitpunktes des Teilprojekts erhoben. Insgesamt nahmen $N=33$ Grundschulen und $N=71$ Lehrkräfte auf freiwilliger Basis an der Erhebung im Sommer 2016 teil. Die Befragung erfolgte mittels digitaler Fragebögen, die spezifisch für die Evaluation des BiSSProgramms im Bereich Lesen an Grundschulen entwickelt wurden. Insgesamt haben 30 Schulleitungen und 41 Lehrkräfte die Fragebögen ausgefüllt zurückgesandt, wobei von den vorliegenden Fragebögen fünf ausgeschlossen werden mussten, da die entsprechenden Personen aufgrund ihrer Funktion nicht zur Stichprobe gehörten. Die weiteren Angaben beruhen daher auf einer Gesamtstichprobe von 66 Personen $(89,4 \%$ weiblich, $7,6 \%$ männlich, $3 \%$ fehlend). Im Hinblick auf das Geschlecht entspricht die Stichprobe der üblichen Verteilung unter Grundschullehrkräften in Deutschland (88,8\% weiblich,
11,2\% männlich; Statistisches Bundesamt, 2016). Auch die Verteilung des Alters (25-29 Jahre: 3\%, 30-39 Jahre: 18\%, 40-49 Jahre: $28 \%$, 50-59 Jahre: 40,9\%, über 60 Jahre: 9,1\%) entspricht in etwa dem bundesweiten Bild der Lehrkräfte allgemein (unter 30 Jahre: 7\%, 3039 Jahre: $26 \%$, 40-49 Jahre: $27 \%$, 50-59 Jahre: $26 \%$, über 60 Jahre: $14 \%$; Statistisches Bundesamt, 2016). Das durchschnittliche Dienstalter (ohne Referendariat) betrug 21.25 Jahre, mit einer großen Spannweite von zwei bis 42 Jahren $(S D=11.2 ; M d n=19.5)$. Die Dauer der Beteiligung am BiSS-Programm lag bei $M=21.6$ Monaten $(S D=9.88$; $M d n=21)$.

Im Rahmen einer Zusammenarbeit mit dem Institut zur Qualitätsentwicklung im Bildungswesen (IQB) wurden an den Projektschulen zudem Testaufgaben zur Lesekompetenz aus dem IQB-Bildungstrend 2016 eingesetzt, sodass für $N=736$ Schülerinnen und Schüler auch Kompetenzdaten vorlagen. Hiervon konnten $N=572$ Schülerinnen und Schüler mit dem vorliegenden Lehrkräftedatensatz verbunden werden. Von fünf betreffenden Schulen lagen keine Schülerdaten vor. Von den übrigen Schulen nahm jeweils eine Klasse an der Erhebung teil, lediglich bei drei Schulen wurden je zwei Klassen erfasst $\left(M_{\text {Schülerzahl pro Klasse }}=\right.$ 15.81).

\section{Erhebungsmethoden}

Die Skala Qualität der Kooperation wurde mittels fünf Items erhoben (adaptiert nach SINUS-Transfer/SINUS an Grundschulen; u. a. Fischer, Dedekind, Rieck, Prenzel \& Köller, 2010; Ostermeier, 2004; Fischer, Döring, Rieck, Trepke, Dalehefte, Menk \& Köller, 2014). Die Lehrkräfte gaben auf einer vierstufigen Likertskala von stimme nicht $z u$ (1) bis stimme voll $z u$ (4) an, wie sie die Zusammenarbeit der Gruppe einschätzen (Beispielitem: „In der Zusammenarbeit mit den Lehrkräften der BiSS-Gruppe an unserer Schule habe ich den Eindruck, dass wir unsere Ziele auch tatsächlich erreichen."). Die Reliabilität dieser Skala lag bei Cronbachs $\alpha=.87$ (Tabelle 1).

Die Häufigkeit der Kooperation wurde über sieben Items erfasst (adaptiert nach PISA 2003; Ramm et al., 2006). Die vierstufige Antwortskala reichte von nie oder fast nie (1) bis zu jeden Tag oder fast jeden Tag (4) (Beispielitem: „Wie oft kooperieren Sie mit anderen Lehrkräften im Kontext von BiSS bei der gemeinsamen Planung ganzer Unterrichtseinheiten oder von Projekten?"). Diese Skala wies eine Reliabilität von Cronbachs $\alpha=.78$ auf.

Die (kommunikativen) Eigenschaften (eigene Entwicklung) wurden mittels sieben Items (Beispielitem: „Ich bin im Kollegium gut vernetzt.") über eine vierstufige Likertskala von stimme nicht $z u$ (1) bis stimme $z u$ (4) erfasst. Die Reliabilität der Skala lag bei Cronbachs $\alpha=.79$. Anhand 
Tabelle 1. Mittelwerte, Standardabweichungen, Reliabilitäten und Interkorrelationen der kommunikativen Aspekte sowie der wahrgenommenen Entwicklung und der Diffusion (Lehrkraftdaten)

\begin{tabular}{|c|c|c|c|c|c|c|c|c|c|c|c|c|c|c|}
\hline & & M & $S D$ & 1 & 2 & 3 & 4 & 5 & 6 & 7 & 8 & 9 & 10 & 11 \\
\hline 1 & Geschlecht & 1.43 & - & - & & & & & & & & & & \\
\hline 2 & Dienstalter & 21.08 & 11.07 & .08 & - & & & & & & & & & \\
\hline 3 & Schulgröße & 20.18 & 9.21 & .06 & .01 & - & & & & & & & & \\
\hline 4 & (Kommunikative) Eigenschaften & 3.42 & 0.47 & -.23 & -.13 & .21 & .82 & & & & & & & \\
\hline 5 & Häufigkeit der Kooperation & 1.80 & 0.50 & .15 & -.06 & .21 & .24 & .78 & & & & & & \\
\hline 6 & Qualität der Kooperation & 3.15 & 0.59 & -.18 & .07 & .09 & .29 & .16 & .87 & & & & & \\
\hline 7 & Zusammensetzung der Projektgruppe & 3.56 & 0.47 & -.04 & -.05 & .26 & .04 & .10 & .24 & .76 & & & & \\
\hline 8 & Mentale Entwicklung & 3.04 & 0.56 & -.14 & -.06 & .24 & $.32 *$ & $.39 * \star$ & .20 & .30 & .87 & & & \\
\hline 9 & Sensible Entwicklung & 2.93 & 0.70 & -.21 & .12 & .16 & $.28 *$ & $.38 * \star$ & .00 & $.32 *$ & $.66^{\star \star \star}$ & .79 & & \\
\hline 10 & Praktische Entwicklung & 3.28 & 0.58 & -.41 & -.23 & .18 & $.31 *$ & .27 & .07 & .12 & 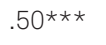 & $.48 * \star$ & .79 & \\
\hline 11 & Diffusion der Veränderung & 3.43 & 0.59 & .31 & .25 & .18 & .19 & .26 & $.34 * \star$ & $.48 * *$ & .27 & $.30 *$ & .09 & .75 \\
\hline
\end{tabular}

Anmerkungen: Kodierung: Geschlecht: 0 (weiblich) bzw. 1 (männlich) (y-standardisiert); Berufsjahre: Anzahl der Jahre im Schuldienst (ohne Referendariat); Schulgröße: Anzahl der Lehrkräfte in der Schule; Pearson Korrelationen; Reliabilitäten (Cronbachs Alpha) kursiv in der Diagonalen; * $p<.05 ; * \star p<.01 ; * \star \star ~ p<.001$, $N_{\text {Lentrkaftebene }}=66, N_{\text {Schulebene }}=33$.

einer explorativen Faktorenanalyse wurde die Skala um ein Item gekürzt, wodurch die Reliablität auf $\alpha=.82$ verbessert werden konnte. Bei der Entwicklung der Skala wurden die Annahmen der Diffusionsforschung zugrunde gelegt, wobei insbesondere die Eigenschaften der Opinion Leaders (Meinungsführer) berücksichtigt wurden (Rogers, 2003; Hall \& Hord, 2006).

Die Zusammensetzung der Projektgruppe (eigene Entwicklung) wurde mittels fünf Items erfasst (Beispielitem: „Die Gruppenmitglieder scheinen eine gute Beziehung zueinander zu haben."). Die vierstufige Antwortskala reichte von stimme nicht $z u$ (1) bis stimme voll zu (4). Die ursprüngliche Skala zeigte eine Reliabilität von Cronbachs $\alpha=.41$, weshalb diese mittels explorativer Faktorenanalyse um zwei Items gekürzt wurde. In der hier verwendeten Version konnte die interne Konsistenz so auf Cronbachs $\alpha=.76$ gesteigert werden. Bei der Entwicklung der Skala wurden die Annahmen und Befunde der Schulentwicklungsforschung zugrunde gelegt, wobei insbesondere die Zusammensetzung von Steuergruppen berücksichtigt wurde, deren Mitglieder nach Rolff (2012b) unter anderem alle bereit sein sollten, sich zusätzlich zu engagieren, sowie aus einer guten Mischung von jüngeren und älteren Lehrkräften bestehen sollte.

Als abhängige Variable wurde die wahrgenommene Entwicklung seit Programmbeginn abgefragt, um einen möglichen Veränderungsprozess zu erfassen (Tabelle 1). Hierzu wurden aus der Skala zur Erhebung der „Wahrgenommenen Entwicklungen durch die Programmarbeit" (adaptiert nach SINUS-Transfer/SINUS an Grundschulen; u. a. Fischer et al., 2010; Ostermeier, 2004; Fischer et al.,
2014) mittels explorativer Faktorenanalyse unter anderem drei neue Subskalen identifiziert: Die mentale Entwicklung, die sensible Entwicklung und die praktische Entwicklung.

Die mentale Entwicklung wurde dabei über fünf Items abgebildet (Beispielitem: „Ich denke systematischer und strukturierter über meinen Leseunterricht nach"), die sensible Entwicklung über zwei Items (Beispielitem: „Ich bin sensibler für Lernschwierigkeiten im Bereich Lesen geworden") und die praktische Entwicklung über vier Items (Beispielitem: „Ich habe neue Inhalte für den Leseunterricht ausprobiert"). Die Lehrkräfte gaben dabei auf einer vierstufigen Likertskala von stimme nicht $z u$ (1) bis stimme voll $z u$ (4) an, wie sie die jeweilige Entwicklung durch die Programmarbeit selbst wahrnehmen. Die Reliabilitäten dieser Skalen lagen zwischen Cronbachs $\alpha=.79$ und .88 (Tabelle 1).

Die Diffusion der Veränderung (adaptiert nach SINUS an Grundschulen; Zentrale Koordinierungsstelle „SINUS an Grundschulen“, 2018) wurde über vier Items erhoben (Beispielitem: „Das BiSS-Projekt ist für die Lehrkräfte an meiner Schule selbstverständlich geworden."). Die vierstufige Antwortskala reichte von stimme nicht zu (1) bis stimme voll zu (4) und die Reliabilität lag bei Cronbachs a $=.69$. Aufgrund einer explorativen Faktorenanalyse wurde für die vorliegenden Analysen ein Item ausgeschlossen, wodurch sich die Reliabilität auf Cronbachs $\alpha=.75$ erhöhte. Bei SINUS an Grundschulen zeigte die Originalskala eine Reliabilität von Cronbachs $\alpha=.74$.

Als Schüleroutcomes wurden die schulischen Kompetenzen im Bereich Lesen herangezogen, die mit Testinstru- 
Tabelle 2. Mittelwerte, Standardabweichungen, Reliabilitäten und Korrelation der Schulgröße, der schulischen Kompetenz im Bereich Lesen (WLE; Schülerdaten) sowie der wahrgenommenen Entwicklung und der Diffusion (Lehrkraftdaten)

\begin{tabular}{|c|c|c|c|c|c|c|c|c|c|}
\hline & & M & $S D$ & 1 & 2 & 3 & 4 & 5 & 6 \\
\hline 1 & Schulgröße & 20.03 & 8.87 & - & & & & & \\
\hline 2 & Schulische Kompetenzen im Bereich Lesen (WLE) & 491.46 & 102.49 & -.10 & .68 & & & & \\
\hline 3 & Mentale Entwicklung & 3.07 & 0.44 & $.44^{\star \star \star}$ & .07 & .87 & & & \\
\hline 4 & Sensible Entwicklung & 2.93 & 0.50 & .24 & .12 & $.81^{\star \star *}$ & .79 & & \\
\hline 5 & Praktische Entwicklung & 3.26 & 0.38 & $.34^{* \star}$ & .01 & $.81 * \star *$ & $.64^{\star \star *}$ & .79 & \\
\hline 6 & Diffusion der Veränderung & 3.41 & 0.57 & $.35^{\star}$ & $.20 \star \star$ & 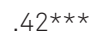 & $.53 * \star \star$ & .24 & .75 \\
\hline
\end{tabular}

Anmerkungen: WLE = Weighted Likelihood Estimates (Schulische Kompetenzen im Bereich Lesen) auf Metrik der Bildungsstandards des Instituts zur Qualitätsentwicklung im Bildungswesen $(M=500, S D=100)$ transformiert; Schulgröße: Anzahl der Lehrkräfte in der Schule; Pearson Korrelation; Reliabilitäten (Cronbachs Alpha bzw. WLE-Reliabilität) kursiv in der Diagonalen; ${ }^{*} p<.05 ; * \star p<.01 ; * \star * p<.001, N_{\text {Schülerebene }}=539, N_{\text {Schulebene }}=26$.

Tabelle 3. Übersicht über die Datenstruktur

\begin{tabular}{|c|c|c|c|c|}
\hline & Datenstruktur & & Modell 1 & Modell 2 \\
\hline Organisationsebene & Schule & & $\begin{array}{l}\text { Schulleitungsangaben; } \\
\text { Aggregierte Lehrkraftangaben }\end{array}$ & $\begin{array}{l}\text { Schulleitungsangaben; } \\
\text { Aggregierte Lehrkraftangaben }\end{array}$ \\
\hline Individuelle Ebene & Lehrkräfte & Schülerinnen und Schüler & Lehrkraftangaben & Schülerangaben \\
\hline
\end{tabular}

menten aus dem IQB-Bildungstrend 2016 erhoben wurden (Tabelle 2). Die Lesekompetenzen wurden dabei über standardisierte Kompetenztests erfasst (für eine detailliertere Beschreibung der Instrumente vgl. BremerichVos, Böhme, Krelle, Weirich \& Köller, 2017). Die Antworten der Schülerinnen und Schüler wurden anschließend vom IQB anhand eindimensionaler Modelle der Item-Response-Theorie (Raschmodell; Embretson \& Reise, 2000) mit dem Paket „TAM“ (Robitzsch, Kiefer \& Wu, 2018) im Statistikprogramm R skaliert. Die daraus resultierenden Schätzer der Personenfähigkeit (Weighted Likelihood Estimates, WLE; Warm, 1989) wurden auf die Metrik der Bildungsstandards $(M=500, S D=100)$ transformiert. Die WLE-Reliabilität lag bei .68, was als akzeptabel angesehen werden kann.

\section{Datenanalyse}

Zur Datenanalyse wurden jeweils die Lehrkraft- und Schülervariablen auf Itemebene ausgewählt, die in das Modell eingehen, und mit SPSS (Version 23) Skalen (Mittelwerte) gebildet. Beim Lehrkraftdatensatz lag der Anteil der fehlenden Skalenwerte dabei zwischen 3 und $42 \%$ pro Skala, beim Schülerdatensatz zwischen 14 und $30 \%$ pro Skala. Alle fehlenden Mittelwerte wurden anschließend mittels Multipler Imputation in Mplus 7.4 (Muthén \& Muthén, 2015) geschätzt. Es wurden jeweils fünfzig Daten- sätze erzeugt, auf deren Basis alle weiteren Analysen mit Mplus gerechnet wurden.

Beim Lehrkraftdatensatz wurden zur Imputation Hilfsvariablen verwendet und die Mehrebenenstruktur berücksichtigt (Lüdtke, Robitzsch \& Grund, 2017). Beim Schülerdatensatz wurden zur Imputation Daten aus Klassenlisten (Geschlecht, Alter, Flucht- und Zuwanderungshintergrund, Deutsch als Muttersprache, Deutsch- und Mathenote) als Hilfsvariablen verwendet, wobei trotzdem 33 Fälle ausgeschlossen werden mussten, da hier alle relevanten Variablen fehlende Werte aufwiesen.

Da die Schülerinnen und Schüler nicht den Lehrkräften, sondern lediglich den Schulen zugeordnet werden konnten, wurden zwei einzelne Modelle gerechnet, wobei im ersten Modell die Lehrkraftangaben (individuelle Ebene) sowie die Schulleitungsangaben und aggregierte Lehrkraftangaben (Organisationsebene) berücksichtig wurden. Im zweiten Modell wurden dann die Schülerangaben (individuelle Ebene) und die Schulleitungsangaben sowie aggregierten Lehrkraftangaben (Organisationsebene) verbunden (Tabelle 3).

\section{Modell 1}

Für die Analyse möglicher Zusammenhänge der kommunikativen Strukturen und Prozesse mit der wahrgenommenen Entwicklung der Betroffenen auf individueller Ebene (Hypothese 1) und als Schulkultur bzw. Arbeitsklima auf Organisationsebene mit der wahrgenommenen Entwicklung sowie der Diffusion der Veränderung ( $\mathrm{Hy}-$ 
pothese $2 \mathrm{a} \& \mathrm{~b}$ ), wurde der Lehrkraftdatensatz anhand eines Mehrebenendesigns in einem multivariaten Modell analysiert. So konnten die Zusammenhänge der Faktoren auf beiden Ebenen simultan untersucht werden. Hierbei wurden die Lehrkräfte auf Level 1 und die Schulen auf Level 2 berücksichtigt, um zu analysieren, welche Merkmale auf welcher Ebene die wichtigsten Prädiktoren sind. Auf Level 1 wurden dabei Regressionen der individuellen Einschätzungen zu den (kommunikativen) Eigenschaften, der Häufigkeit und der Qualität der Kooperation auf die wahrgenommenen Entwicklungen betrachtet, wobei die Variablen am Gesamtmittelwert zentriert wurden, was dem Standardvorgehen bei der Berechnung von Kontexteffekten entspricht (Lüdtke, Marsh, Robitzsch, Trautwein, Asparouhov \& Muthén, 2008; Raudenbush \& Bryk, 2002). Bei den Analysen wurden zudem wie üblich demographische Kontrollvariablen (Geschlecht und Dienstalter) berücksichtigt (vgl. z. B. Rogiest, Segers \& Witteloostuijn, 2015). Auf Level 2 wurden Regressionen der manifesten Clustermittelwerte der Level-1-Variablen sowie der Zusammensetzung der Projektgruppe (Level-2Variable, Angabe der Schulleitung für die Schule) auf die wahrgenommene Entwicklung sowie die Diffusion der Veränderung untersucht, um den Einfluss der Variablen auf dieser Ebene zu überprüfen. Da es sich bei der Diffusion der Veränderung um eine Level-2-Variable handelt, wurde diese lediglich auf der Organisationsebene überprüft. Auf Level 2 wurde bei den Analysen für die Schulgröße - operationalisiert über die Anzahl der Lehrkräfte kontrolliert. Damit wurden in den Analysen auch auf Organisationsebene zusätzliche Informationen der entsprechenden Ebene berücksichtig, die für die Effekte bedeutsam sein können (van Dick et al., 2005). So könnten Lehrkräfte in größeren Schulen eher durch kooperative Strukturen profitieren, da die Vielzahl von schulischen Aufgaben auf Organisationsebene auf mehrere Kolleginnen und Kollegen verteilt werden können. Darüber hinaus wurde hierdurch ein globaler Indikator für die generellen Strukturen und Prozesse an den Einzelschulen berücksichtigt.

Aufgrund der Stichprobengröße ist die Clustergröße im Lehrkraftdatensatz zumeist auf die Schulleitung sowie eine Lehrkraft begrenzt $\left(M_{\text {Clustergröße }}=2\right)$, wobei sechs Cluster $n=1$, 23 Cluster $n=2$, drei Cluster $n=3$ und ein Cluster $n=5$ Personen aufwiesen.

\section{Modell 2}

Um Zusammenhänge der wahrgenommenen Entwicklung der Betroffenen sowie der Diffusion der Verände- rung mit den Schülerkompetenzen im Bereich Lesen zu prüfen (Hypothese $3 \mathrm{a} \& \mathrm{~b}$ ), wurde ein weiteres Mehrebenenmodell auf Grundlage des Schülerdatensatzes - verbunden mit den Lehrkraftangaben - überprüft. Hierbei wurden auf Level 1 Regressionen des Geschlechts, der Muttersprache und des Migrationshintergrunds auf die Lesekompetenz betrachtet, welche übliche Prädiktoren für die Schülerleistung darstellen (vgl. z. B. Tarelli, Valtin, Bos, Bremerich-Vos \& Schwippert, 2012; Segerer, Marx, Stanat, Schneider, Roick \& Marx, 2013). Auf Level 2 wurden Regressionen der mentalen, sensiblen und praktischen Entwicklung (aggregiert auf Schulebene) sowie der Diffusion der Veränderung geprüft, wobei analog zu dem vorherigen Modell auf dieser Ebene für die Schulgröße kontrolliert wurde.

\section{Ergebnisse}

Im Rahmen des ersten Mehrebenenmodells wurden die mentale Entwicklung, die sensible Entwicklung und die praktische Entwicklung sowie die Diffusion der Veränderung als abhängige Variablen berücksichtigt. Die Intraklassenkoeffizienten für die drei Subskalen der wahrgenommenen Entwicklung weisen Werte von $\rho_{\text {IC }}=.23$ bis .45 auf. Die Intraklassenkorrelation (ICC) ist dabei definiert als das Verhältnis der Varianz zwischen den Clustern (Level-2-Varianz) zur Gesamtvarianz (Geiser, 2011). Somit können 23 bis $45 \%$ der Varianz in den wahrgenommenen Entwicklungen der Betroffenen auf Unterschiede zwischen den Schulen zurückgeführt werden. Es erscheint daher sinnvoll, Prädiktorvariablen auf Organisationsebene (Level 2) zu betrachten (Geiser, 2011).

Im Hinblick auf die Überprüfung von Zusammenhängen der kommunikativen Strukturen und Prozesse auf individueller Ebene mit der wahrgenommene Entwicklung der Betroffenen (Hypothese 1) zeigen die Ergebnisse (Tabelle 4), dass die (kommunikativen) Eigenschaften die sensible Entwicklung positiv bedingen $(\beta=0.36 ; p<.05)$. Darüber hinaus sagt die Häufigkeit der Kooperation die mentale $(\beta=0.44 ; p<.05)$ und die sensible Entwicklung ( $\beta=0.44 ; p<.01)$ positiv vorher. Die Qualität der Kooperation bedingt die sensible Entwicklung hingegen negativ $(\beta=-0.33 ; p<.05)$. Auf individueller Ebene können die kommunikativen Strukturen und Prozesse zwischen 33 und $49 \%$ der Varianz aufklären (Tabelle 4). ${ }^{2}$ Entsprechend wird die Hypothese, dass kommunikative Strukturen und Prozesse auf individueller Ebene positiv prädiktiv

\footnotetext{
Aufgrund einer Forderung der Gutachterinnen und Gutachter haben wir das Modell zusätzlich ohne Kontrollvariablen gerechnet, wobei sich auf individueller Ebene ein $R^{2}$ zwischen .25 und .43 und auf Organisationsebene ein $R^{2}$ zwischen .40 und .54 gezeigt hat.
} 
Tabelle 4. Kommunikative Aspekte als Prädiktoren von selbstberichteter mentale, sensibler und praktischer Entwicklung sowie Diffusion der Veränderung

\begin{tabular}{|c|c|c|c|c|c|c|c|c|}
\hline & \multicolumn{2}{|c|}{$\begin{array}{c}\text { Mentale } \\
\text { Entwicklung }\end{array}$} & \multicolumn{2}{|c|}{$\begin{array}{c}\text { Sensible } \\
\text { Entwicklung }\end{array}$} & \multicolumn{2}{|c|}{$\begin{array}{l}\text { Praktische } \\
\text { Entwicklung }\end{array}$} & \multicolumn{2}{|c|}{$\begin{array}{l}\text { Diffusion der } \\
\text { Veränderung }\end{array}$} \\
\hline & $\beta$ & $S E$ & $\beta$ & $S E$ & $\beta$ & $S E$ & $\beta$ & SE \\
\hline \multicolumn{9}{|l|}{ Lehrkraftebene } \\
\hline Geschlecht & -0.08 & 0.17 & -0.14 & 0.14 & -0.23 & 0.17 & & \\
\hline Dienstalter & -0.05 & 0.19 & 0.19 & 0.19 & -0.18 & 0.19 & & \\
\hline (Kommunikative) Eigenschaften & 0.34 & 0.23 & $0.36^{\star}$ & 0.22 & 0.30 & 0.23 & & \\
\hline Häufigkeit der Kooperation & $0.44^{\star *}$ & 0.22 & $0.44^{\star}$ & 0.24 & 0.10 & 0.29 & & \\
\hline Qualität der Kooperation & -0.07 & 0.20 & $-0.33 *$ & 0.18 & -0.10 & 0.20 & & \\
\hline$R^{2}$ & & .47 & & .49 & & .33 & & \\
\hline \multicolumn{9}{|l|}{ Schulebene } \\
\hline Schulgröße & 0.19 & 0.37 & -0.04 & 0.30 & 0.20 & 0.43 & -0.03 & 0.19 \\
\hline (Kommunikative) Eigenschaften & -0.23 & 0.44 & -0.12 & 0.43 & -0.19 & 0.59 & 0.02 & 0.22 \\
\hline Häufigkeit der Kooperation & -0.23 & 0.48 & -0.09 & 0.46 & 0.26 & 0.59 & 0.19 & 0.19 \\
\hline Qualität der Kooperation & 0.28 & 0.37 & 0.12 & 0.35 & 0.08 & 0.48 & 0.34 & 0.22 \\
\hline Zusammensetzung der Projektgruppe & 0.30 & 0.36 & $0.55^{\star}$ & 0.29 & 0.14 & 0.40 & $0.30 *$ & 0.18 \\
\hline$R^{2}$ & & .57 & & .61 & & .55 & & .41 \\
\hline ICC & \multicolumn{2}{|c|}{.45} & \multicolumn{2}{|c|}{.23} & \multicolumn{2}{|c|}{.34} & & \\
\hline
\end{tabular}

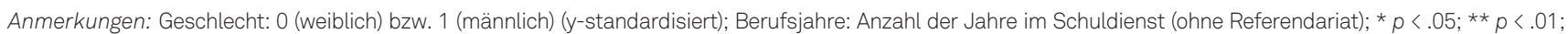

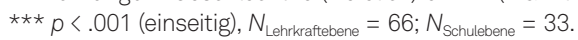

für die mentale, sensible und praktische Entwicklung der Betroffenen sind, von den Daten gestützt. Lediglich der negative Effekt der Qualität der Kooperation muss weiter geprüft werden.

Im Hinblick auf die Überprüfung von Zusammenhängen der kommunikativen Strukturen und Prozesse als Schulkultur bzw. Arbeitsklima auf Organisationsebene mit der wahrgenommene Entwicklung der Betroffenen (Hypothese 2a) sowie die Diffusion der Veränderung (Hypothese 2b) zeigen die Ergebnisse (Tabelle 4), dass lediglich die Zusammensetzung der Projektgruppe die sensible Entwicklung $(\beta=0.55 ; p<.05)$ sowie die Diffusion der Veränderung ( $\beta=0.30 ; p<.05)$ positiv bedingt. Auf Organisationsebene können die kommunikativen Strukturen und Prozesse zwischen 41 und $61 \%$ der Varianz aufklären. Die Daten sprechen folglich für die Hypothese, dass (a) kommunikativen Strukturen und Prozesse auf Organisationsebene positiv prädiktiv für die Diffusion der Veränderung sind. Die Hypothese, dass (b) die kommunikativen Strukturen und Prozesse auch positiv prädiktiv für die mentale, sensible und praktische Entwicklung der Betroffenen sind, wurde - abgesehen im Hinblick auf die sensible Entwicklung - hingegen nicht gestützt.

Die Überprüfung von Zusammenhängen der wahrgenommenen Entwicklung der Betroffenen sowie der Diffusion der Veränderung mit den Schülerkompetenzen im Bereich Lesen (Hypothese $3 \mathrm{a} \& \mathrm{~b}$ ) zeigte, dass lediglich
Tabelle 5. Wahrgenommene Entwicklung sowie Diffusion der Veränderung als Prädiktoren für die schulischen Kompetenzen im Bereich Lesen (WLE)

\begin{tabular}{lll}
\hline & \multicolumn{2}{c}{ WLE } \\
& $\beta$ & $S E$ \\
\hline Schülerebene & & \\
Geschlecht & $0.09^{*}$ & 0.05 \\
Deutsch als Muttersprache & $-0.23^{* * *}$ & 0.07 \\
Migrationshintergrund & -0.07 & 0.05 \\
\hline$R^{2}$ & .09 & \\
\hline Schulebene & & \\
Schulgröße & -0.13 & 0.22 \\
Mentale Entwicklung & 0.12 & 0.42 \\
Sensible Entwicklung & 0.02 & 0.38 \\
Praktische Entwicklung & -0.19 & 0.33 \\
Diffusion der Veränderung & $0.68 * * *$ & 0.18 \\
\hline$R^{2}$ & .50 & \\
\hline ICC & .12 \\
\hline
\end{tabular}

Anmerkungen: WLE = Weighted Likelihood Estimates (Schulische Kompetenzen im Bereich Lesen); Kodierung: WLE: auf Metrik der Bildungsstandards des Instituts zur Qualitätsentwicklung im Bildungswesen $(M=500, S D=100)$ transformiert; Geschlecht: $0=$ männlich, 1 = weiblich (y-standardisiert); Deutsch als Erstsprache: 1 = ja, 2 = nein (y-standardisiert); Migrationshintergrund: 0 = kein Migrationshintergrund; 1 = ein Elternteil im Ausland geboren, 2 $=$ zweite Generation; 3 = erste Generation; $* p<.05 ; * \star p<.01 ; * \star \star ~ p<.001$ (einseitig), $N_{\text {Schülerebene }}=539 ; N_{\text {Schulebene }}=26$. 
die Diffusion der Veränderung auf Organisationsebene positiv prädiktiv für die schulischen Kompetenzen im Bereich Lesen auf Seiten der Schülerinnen und Schüler ist ( $\beta$ $=0.68 ; p<.001)$. Die wahrgenommene Entwicklung der Beteiligten sowie die Diffusion der Veränderung klären auf Organisationsebene dabei $50 \%$ der Varianz auf. ${ }^{3}$ Auf individueller Ebene sagen das Geschlecht und die Muttersprache die Lesekompetenz vorher, wobei die hier betrachteten Prädiktoren 9\% der Varianz auf individueller Ebene aufklären. Die ICC fiel in diesem Modell mit $\rho_{\mathrm{IC}}=$ .12 erwartungsgemäß gering aus, da sich die Kompetenzen vor allem auf individueller Ebene der Schülerinnen und Schüler entscheiden. Entsprechend wird die Hypothese, dass die Diffusion der Veränderung positiv prädiktiv für die Schülerkompetenzen im Bereich Lesen ist (b), von den Daten gestützt. Die Hypothese, dass auch die mentale, sensible und praktische Entwicklung positiv prädiktiv für die Schülerkompetenzen sind (a) wird hingegen nicht gestützt.

\section{Diskussion}

Die vorliegende Studie hatte zum Ziel, die individuellen und organisationalen Prozesse und Strukturen an den Einzelschulen besser zu verstehen - vor allem um solche zu identifizieren, die einen entsprechenden Entwicklungsprozess auf Seiten der Lehrkräfte und schlussendlich der Schülerinnen und Schüler fördern können.

Anhand der vorliegenden Daten aus dem Evaluationsprojekt BiSS-EvalLesen konnte gezeigt werden, dass auf individueller Ebene insbesondere die Häufigkeit der Kooperation positiv prädiktiv für die wahrgenommene Entwicklung der Beteiligten in einem schulischen Implementationsprozess ist. Beteiligte, die also berichten, eine häufige Kooperation mit ihren Kolleginnen und Kollegen wahrzunehmen, stellen bei sich selbst auch eine stärkere Entwicklung seit Programmbeginn fest. Hier kann angenommen werden, dass durch entsprechend häufige Rückmeldungen von Kolleginnen und Kollegen die Entwicklungsprozesse reflektiert und vorangetrieben werden können. Darüber hinaus kann auch die kooperative Unterstützung positiv auf die Entwicklung wirken. Generell kann die Relevanz von Kooperation in schulischen Implementationsprozessen somit bestätigt werden (Steinert et al., 2006; Borko, 2004; Gräsel et al., 2006; Jäger, 2004; Goldenbaum, 2013).
Darüber hinaus sagen die (kommunikativen) Eigenschaften die sensible Entwicklung positiv vorher. Denken die Beteiligten also unter anderem über sich selbst, dass sie sich gut mitteilen können, gut im Kollegium vernetzt sind und eine hohe Glaubwürdigkeit im Kollegium genießen, so nehmen sie seit Programmbeginn eine verstärkte sensible Entwicklung bei sich wahr.

Die Qualität der Kooperation bedingt die sensible Entwicklung entgegen der Erwartungen hingegen negativ. ${ }^{4}$ Berichten die Beteiligten also unter anderem davon, dass innerhalb der Gruppe alle an einem Strang ziehen, die Arbeitsteilung gut gelingt und die Ziele auch tatsächlich erreicht werden, so nehmen sie eine geringere sensible Entwicklung bei sich wahr. Dieser Befund weist insbesondere daraufhin, dass innerhalb des Konstrukts der Kooperation differenziert werden muss, um entsprechende Zusammenhänge aufzudecken (vgl. Steinert et al., 2006). Es kann vermutet werden, dass eine qualitativ hochwertige Kooperation die individuelle Lehrkraft entlastet, sodass diese sich entsprechend weniger mit den Maßnahmen und der eigenen Entwicklung auseinandersetzt.

Auf Organisationseben hat sich anhand der Daten hingegen gezeigt, dass die kooperativen Strukturen und Prozesse - im Sinne der Schulkultur bzw. des Arbeitsklimas nicht prädiktiv für die wahrgenommene Entwicklung der Beteiligten in einem schulischen Implementationsprozess sind. Auf Organisationseben bedingt lediglich die Zusammensetzung der Projektgruppe die sensible Entwicklung sowie die Diffusion der Veränderung. Sind die Mitglieder der Gruppe also alle bereit, sich zu engagieren, besteht die Gruppe aus jüngeren und älteren Lehrkräften und scheinen diese eine gute Beziehung zueinander zu haben, so nehmen die einzelnen Lehrkräfte eine verstärkte sensible Entwicklung bei sich wahr und die Maßnahmen sind innerhalb der Schule verbreiteter. Anhand der vorliegenden Daten konnte somit auch die Relevanz des Teams für erfolgreiche Implementationsprozesse bestätigt werden (Argote et al., 2003; Zacher et al., 2008).

Im Hinblick auf die Schülerdaten hat sich zudem gezeigt, dass die Diffusion der Veränderung zwei Jahre nach dem Beginn der Programmarbeit im Rahmen von BiSS auf Organisationsebene der stärkste Prädiktor für die Kompetenz der Schülerinnen und Schüler im Bereich Lesen ist. Die wahrgenommene Entwicklung der Beteiligten seit Programmbeginn scheint hier keine statistisch bedeutsame Vorhersagekraft zu besitzen. Berichtet die Schulleitung also davon, dass das Programm in der eigenen Schule weit verbreitet ist, so schneiden die Schüle-

\footnotetext{
Ohne Kontrolle der Schulgröße $R^{2}=.49$.

Der negative Effekt verschwindet jedoch, wenn die (kommunikativen) Eigenschaften aus dem Modell entfernt werden.
} 
rinnen und Schüler dieser Schule besser im Kompetenztest ab. Diese Befunde erscheinen im Sinne des Evaluationsprojekts BiSS-EvalLesen erfreulich, da es sich bei dem entsprechenden Bereich des BiSS-Programms um eine fachübergreifende Diagnose und Förderung im Bereich Lesen handelt und die Schülerinnen und Schüler somit bestenfalls durch alle Lehrkräfte einer Schule - sowohl im Deutsch- als auch im Fach- oder Vertretungsunterricht in Kontakt mit den Programmmaßnahmen kommen und gefördert werden. Da die Schulen entsprechend der genannten Module das Ziel hatten, durch die Teilnahme an BiSS die Diagnose und Förderung der Leseflüssigkeit und ihrer Voraussetzungen sowie die Diagnose und Förderung des Leseverständnisses zu verbessern, erscheinen die Kompetenzunterschiede zwischen Schulen, in denen die BiSS-Maßnahmen verbreiteter bzw. weniger verbreitet sind, ein Indikator für einen erfolgreichen Implementationsprozess. Schlussendlich kann das BiSS-Programm somit auch zu einem besseren Abschneiden in Schulleistungsstudien beitragen - wenn die Maßnahmen in den jeweiligen Schulen verbreitet sind.

\section{Implikationen}

Ziel der vorliegenden Studie war es zudem, konkrete Hinweise zu formulieren, sodass bestehende und künftige Programme eine angestrebte Veränderung auf Seiten der Lehrkräfte sowie schlussendlich eine tatsächliche Verbesserung der Kompetenzen auf Seiten der Schülerinnen und Schüler fördern können.

Auf Grundlage der vorliegenden Daten kann festgehalten werden, dass Change Management für die Organisation Schule auf individueller Ebene vor allem bedeutet, diejenigen, die die Neuerung hauptverantwortlich umsetzen sollen, sorgfältig und im Hinblick auf bestimmte Aspekte auszuwählen sowie entsprechende kooperative Strukturen bereitzustellen, um eine häufige Kooperationen zu ermöglichen. Dies kann sich im Hinblick auf die individuelle Ebene als förderlich für die Entwicklung der Beteiligten erweisen. Auf Organisationseben kann im Rahmen des Change Managements vor allem an der Projektgruppe angesetzt werden, die sowohl die sensible Entwicklung als auch die Diffusion der Veränderung in der Schule bedingt, wobei letztere sich bereits nach kurzer Implementationsdauer in den Kompetenzen der Schülerinnen und Schüler niederzuschlagen scheint.

Laut Rolff (2012a) sind neben Change Management auch Organisations-, Unterrichts- und Personalentwicklung Komponenten der Schulentwicklung, die als Synthese wirken. Entsprechend kann im Rahmen eines Implementationsprozess an allen drei Komponenten angesetzt werden. Aufgrund der Erkenntnisse aus der Evaluation können so entsprechende Hinweise abgeleitet werden, anhand derer das Change Management wirksam werden kann.

Im Sinne der Organisationsentwicklung sollten sich insbesondere die an BiSs beteiligten Schulen, aber auch Schulen, die vergleichbare Implementationsprozesse planen bzw. umsetzen, somit um die Einrichtung einer Projektgruppe bemühen, die die Diffusion der Veränderung vorantreibt. Im Sinne des Change Managements kann eine solche Einrichtung von oben vorgegeben werden, wobei jedoch entsprechende Richtlinien mitgegeben werden sollten, sodass die Beteiligten vor Ort im Sinne der Organisationsentwicklung die Weiterentwicklung der Organisation von innen heraus und durch deren Mitglieder selbst - aber in vorgegebenem Rahmen - vorantreiben können. Die Wirksamkeit der Projektgruppe kann dann wieder im Rahmen des Change Management mittels Evaluation überprüft werden. Hier würde somit wieder eine Kombination aus Top-down- und Bottom-Up-Strategie stattfinden. Dabei kann die Personalentwicklung unterstützend wirken, indem diese die Teammitglieder systematisch auswählen und sowohl die einzelnen Mitglieder als auch das ganze Team entwickeln kann. So können Teamentwicklung und -trainings langfristig das Wissen und die Fertigkeiten der einzelnen Teammitglieder verbessern (Zacher et al., 2008). Auch zur Entwicklung des ganzen Teams stehen verschiedene Teamentwicklungsmaßnahmen zur Verfügung (vgl. Philipp, 2006). Das entsprechende Klima in Teams können Verantwortliche dabei zum Beispiel über das Teamklima-Inventar (Brodbeck \& Maier, 2001) erfassen.

Darüber hinaus kann das Change Management im Hinblick auf die Organisationsentwicklung verstärkt dazu anregen kooperative Strukturen und Prozesse in den Einzelschulen zu etablieren, indem räumliche und zeitliche Kapazitäten geschaffen werden. So könnte die Entwicklung der Beteiligten im Implementationsprozess gefördert werden. Hier kann zudem erneut auch die Personalentwicklung unterstützend wirken, da diese die Beteiligten bei den Herausforderungen, die Kooperation mit sich bringt - vor allem auch vor dem Hintergrund des „Autonomie-Paritätsmusters“ -, begleiten kann. So kann die Personalentwicklung die Beteiligten dazu bestärken, ihren Unterricht zu öffnen und sich mit Kolleginnen und Kollegen auszutauschen. Im Rahmen von individuellen Trainings könnten zudem auch die (kommunikativen) Eigenschaften der Beteiligten gefördert werden.

\section{Limitationen}

Die Generalisierbarkeit der aufgezeigten Ergebnisse ist aufgrund des geringen Umfangs der Stichprobe und des 
querschnittlichen Designs limitiert. Eine größere Stichprobe zur besseren Absicherung der Ergebnisse wäre wünschenswert gewesen. Die berichteten Erkenntnisse sind entsprechend mit künftiger Forschung abzusichern und weiter auszudifferenzieren. Da relativ viele angenommene Zusammenhänge nicht signifikant geworden sind, ist vor allem zu prüfen, ob anhand einer größeren Stichprobe gegebenenfalls weitere Effekte aufgedeckt werden können. Hierbei könnten auch methodische Neuerungen auf dem Gebiet der Mehrebenenanalyse Anwendung finden, die speziell für kleine bis mittelgroße Stichproben entwickelt wurden (z. B. Zitzmann, 2018).

Zudem hätten längsschnittliche Daten - vor allem im Hinblick auf die wahrgenommene Entwicklung - die Interpretation der Befunde gestärkt. Darüber hinaus wurden die abhängigen Variablen im ersten Modell lediglich aufgrund der selbstberichteten Wahrnehmung der beteiligten Lehrkräfte bzw. Schulleitungen erfasst, weshalb generelle Einschränkungen im Hinblick auf die Reliabilität und die Validität berücksichtigt werden müssen (McGraw, Sellers, Stone, Resnicow, Kuester, Fridinger \& Wechsler, 2000). Da die Schülerinnen und Schüler lediglich den Schulen und nicht den einzelnen Lehrkräften zugeordnet werden konnten, wurden die Lehrkraft- und Schülerdaten auf Schulebene verbunden (Modell 2), wodurch die Varianz innerhalb einer Schule entsprechend vernachlässigt wurde (Schulmittelwerte). Ebenfalls aufgrund der Datenstruktur konnten lediglich zwei einzelne Zwei-Ebenen-Modelle gerechnet werden. Die Annahmen in einem einzigen Drei-Ebenen-Modell prüfen zu können, wäre wünschenswert gewesen, bleibt jedoch Aufgabe künftiger Forschungsvorhaben. Darüber hinaus wäre es spannend, dem theoretischen Wirkmodell entsprechende Mediationseffekte zu überprüfen.

\section{Fazit}

Die vorliegende Studie konnte anhand eines Mehrebendesigns simultan kommunikative Strukturen und Prozesse auf individueller und organisationaler Ebene in einem schulischen Implementationsprozess prüfen. Auf Grundlage der Ergebnisse konnten Hinweise formuliert werden, wie im Rahmen des Change Managements bestehende Implementationsprozesse - im Hinblick auf BiSS, aber auch darüber hinaus - sowie künftige Bestrebungen optimiert werden könnten. Dabei können im Sinne des Change Managements entsprechende Richtlinien vorgegeben, im Rahmen von Organisations- und Personalentwicklung vor Ort umgesetzt, so Unterrichtsentwicklung gefördert und schlussendlich im Rahmen des Change Managements auch wieder überprüft werden.
Die berichteten Befunde sind jedoch nicht nur für Schulen relevant, sondern vor allem auch für Organisationen, die schulähnliche Strukturen - besonders im Hinblick auf die Mitarbeiterinnen und Mitarbeiter (Personalauswahl, Aufgaben der Führungskraft, Macht- und Einflussfaktoren; Weishart, 2008) - aufweisen. So erscheinen die Befunde zu den kommunikativen Strukturen und Prozesse im Implementationsprozess zum Beispiel auf Universitäten, Krankenhäuser und Gerichte übertragbar, da diese organisationstheoretisch mit Schulen verwandt sind (vgl. Weishart, 2008).

Auch wenn die schulischen Strukturen berücksichtigt werden müssen, erscheint ein Vergleich von Schule mit anderen Organisationen fruchtbar, in deren Forschungsfeldern bereits zahlreiche Hinweise zu (kommunikativen) Faktoren im Change Management vorliegen (vgl. z. B. medizinischer Bereich). Darüber hinaus kann es die Aufgabe künftiger Forschung sein, die konstatierten Befunde im Rahmen von ganz anderen Implementationsprozessen in Schulen zu replizieren (vgl. Teerling \& Köller, 2019).

\section{Literatur}

Altrichter, H. \& Eder, F. (2004), Das „Autonomie-Paritätsmuster“ als Innovationsbarriere? In H.-G. Holtappels (Hrsg.), Schulprogramme - Instrumente der Schulentwicklung. Konzeptionen, Forschungsergebnisse, Praxisempfehlungen (S. 195-221). Weinheim \& München: Juventa Verlag.

Argote, L., McEvily, B. \& Reagans, R. (2003). Managing Knowledge in Organizations: An Intergrative Framework and Review of Emerging Themes. Management Science, 49, 571-582.

Batinic, B., Haupt, C. M. \& Wieselhuber, J. (2006). Validierung und Normierung des Fragebogens zur Erfassung von Trendsetting (TDS). Diagnostica, 52, 60-72. https://doi.org/10.1026/00121924.52.2.60

Baumert, J., Klieme, E., Neubrand, M., Prenzel, M., Schiefele, U., Schneider, W. et al. (2001). PISA 2000. Basiskompetenzen von Schülerinnen und Schülern im internationalen Vergleich. Opladen: Leske + Budrich.

Becker-Mrotzek, M., Hasselhorn, M., Roth, H.-J., Stanat, P. (2016). Die Bund-Länder Initiative „Bildung durch Sprache und Schrift (BISS)“. Psychologie in Erziehung und Unterricht, 63, 75-77. https://doi.org/10.2378/peu2016.art07d

Bergmann, K. \& Rollett, W. (2008). Kooperation und kollegialer Konsens bzw. Zusammenhalt als Bedingungen der Innovationsbereitschaft von Lehrerkollegien in Ganztagsschulen. In: E.M. Lankes (Hrsg.), Pädagogische Professionalität als Gegenstand empirischer Forschung (S. 291-301). Münster: Waxmann.

Borko, H. (2004). Professional Development and Teacher Learning: Mapping the Terrain. Educational Researcher, 33 (8), $3-15$ https://doi.org/10.3102/0013189X033008003

Bremerich-Vos, A., Böhme, K., Krelle, M., Weirich, S., \& Köller, O. (2017). Kompetenzstufenmodelle im Fach Deutsch. In P. Stanat, S. Schipolowski, C. Rjosk, S. Weirich, \& N. Haag (Hrsg.), IQB-Bildungstrend 2016. Kompetenzen in den Fächern Deutsch und Mathematik am Ende der 4. Jahrgangsstufe im zweiten Ländervergleich (S. 53 -70). Münster: Waxmann. 
Brodbeck, F. C. \& Maier, G. W. (2001). Das Teamklima-Inventar (TKI) für Innovation in Gruppen. Psychometrische Überprüfung an einer deutschen Stichprobe. Zeitschrift für Arbeits- und Organisationspsychologie, 45, 59-73. https://doi.org/10.1026// 0932-4089.45.2.59

Dick, R. van, Wagner, U., Stellmacher, J. \& Christ, O. (2005). Mehrebenenanalysen in der Organisationspsychologie. Ein Plädoyer und ein Beispiel. Zeitschrift für Arbeits- und Organisationspsychologie, 49, 27 - 34. https://doi.org/10.1026/0932-4089.49.1.27

Edmondson, A. C., Bohmer, R. M. \& Pisano, G. P. (2001). Disrupted Routines: Team Learning and New Technology Implementation in Hospitals. Administrative Science Quarterly, 46, 685- 716.

Embretson, S. E., \& Reise, S. P. (2000). Item response theory for psychologists. Erlbaum: Hillsdale, NJ.

Fischer, C., Dedekind, B., Rieck, K., Prenzel, M. \& Köller, O. (2010), Modellversuchsprogramm SINUS-Transfer Grundschule "Weiterentwicklung des mathematischen und naturwissenschaftlichen Unterrichts an Grundschulen". Abschlussbericht 2004-2009 (Abschlussbericht). Kiel: Leibniz-Institut für die Pädagogik der Naturwissenschaften und Mathematik.

Fischer, C., Döring, B., Rieck, K., Trepke, F., Dalehefte, I.-M., Menk, M. \& Köller, O. (2014). SINUS an Grundschulen. Abschlussbericht. 1. August 2009 bis 31. Juli 2013 (Abschlussbericht). Kiel: Leibniz-Institut für die Pädagogik der Naturwissenschaften und Mathematik.

Fischer, C., Rieck, K. \& Döhring, B. (2019). Implementation eines Ansatzes zur Weiterentwicklung des mathematischen und naturwissenschaftlichen Unterrichts. Erfahrungen aus dem Programm „SINUS an Grundschulen“. Psychologie in Erziehung und Unterricht, 66 (1), 6-18.

Geiser, C. (2011). Datenanalyse mit Mplus. Eine anwendungsorientierte Einführung (2., durchgesehene Auflage). Wiesbaden: VS Verlag für Sozialwissenschaften.

Goldenbaum, A. (2013). Implementation von Schulinnovationen. In M. Rürup \& I. Bormann (Hrsg.), Innovationen im Bildungswesen. Analytische Zugänge und empirische Befunde (S. 149-172). Wiesbaden: Springer VS. https://doi.org/10.1007/978-3-53119701-2_7

Gräsel, C., Jäger, M. \& Wilke, H. (2006). Konzeption einer übergreifenden Transferforschung. In R. Nickolaus \& C. Gräsel (Hrsg.), Innovation und Transfer (S. 445 -566). Baltmannsweiler: Schneider Verlag Hohengehren.

Hall, G. E. \& Hord, S. M. (2006). Implementing Change. Patterns, Principles, and Potholes. Boston: Allyn \& Bacon.

Jäger, M. (2004). Transfer in Schulentwicklungsprojekten. Wiesbaden: VS Verlag für Sozialwissenschaften.

Langer, W. (2009). Mehrebenenanalyse. Eine Einführung für Forschung und Praxis. Wiesbaden: VS Verlag für Sozialwissenschaften.

Lüdtke, O., Marsh, H.W., Robitzsch, A., Trautwein, U., Asparouhov, T. \& Muthén, B. (2008). The multilevel latent covariate model: A new, more reliable approach to group-level effects in contextual studies. Psychological Methods, 13, $203-229$.

Lüdtke, O., Robitzsch, A., \& Grund, S. (2017). Multiple imputation of missing data in multilevel designs: A comparison of different strategies. Psychological Methods, 22 (1), 141 -165. https://doi. org/10.1037/met0000096.supp

McElvany, N., Ohle-Peters, A., Igler, J., Schlitter, T., Teerling, A., Asseburg, R. \& Köller, O. (2018). Evaluation der Leseförderung an Grundschulen im Rahmen von „Bildung durch Sprache und Schrift" (BiSS). In F. Schwabe, N. McElvany, W. Bos \& H. G. Holtappels (Hrsg.), Schule und Unterricht in gesellschaftlicher Heterogenität (Jahrbuch der Schulentwicklung, 20, S. 258280). Weinheim: Beltz.

McGraw, S. A., Sellers, D., Stone, E., Resnicow, K. A., Kuester, S., Fridinger, F. \& Wechsler, H. (2000). Measuring implementation of school programs and policies to promote healthy eating and physical activity among youth. Preventive Medicine, 31, 86-97. https://doi.org/10.1006/pmed.2000.0648

Muthén, B. \& Muthén, L. (2015). Mplus (Version 7.4) [Computer software]. Los Angeles, CA.

Nerdinger, F.W. (2019). Organisationsentwicklung. In F. W. Nerdinger, G. Blickle \& N. Schaper (Hrsg.), Arbeits- und Organisationspsychologie (4., voll. über. Aufl., S. 179 -191). Berlin: Springer.

Ostermeier, C. (2004). Kooperative Qualitätsentwicklung in Schulnetzwerken. Münster: Waxmann.

Philipp, E. (2006). Teamentwicklung in der Schule: Konzepte und Methoden (4. Auflage). Weinheim: Beltz.

Ramm, G., Prenzel, M., Baumert, J., Blum, W., Lehmann, R., Leutner, D. et al. (2006). PISA 2003. Dokumentation der Erhebungsinstrumente. Münster: Waxmann.

Raudenbush, S. W. \& Bryk, A. S. (2002). Hierarchical linear models (2nd ed.). Thousand Oaks, CA: Sage.

Robitzsch, A., Kiefer, T., \& Wu, M. (2018). TAM: Test analysis modules. R package version 2.12-18. https://CRAN.R-project.org/ package =TAM

Rogers, E. M. (2003). Diffusion of Innovations (5th ed.). New York: Free Press.

Rogiest, S., Segers, J. \& Witteloostuijn, A. van (2015). Climate, communication and participation impacting commitment to change. Journal of Organizational Change Management, 28, 1094-1106. https://doi.org/10.1108/JOCM-06-2015-0101

Rolff, H.-G. (2007). Schulentwicklung. In H.E. Tenorth \& R. Tippelt (Hrsg.), Lexikon Pädagogik (S. 622-625). Weinheim \& Basel: Beltz.

Rolff, H.-G. (2012a). Grundlagen der Schulentwicklung. In C. G. Buhren \& H.-G. Rolff (Hrsg.), Handbuch Schulentwicklung und Schulentwicklungsberatung. (S. 12 -24). Weinheim Basel: Beltz.

Rolff, H.-G. (2012b). Steuergruppen und interne Begleitung. In C. G. Buhren \& H.-G. Rolff (Hrsg.), Handbuch Schulentwicklung und Schulentwicklungsberatung. (S. 71 -89). Weinheim Basel: Beltz.

Rosenstiel, L. von \& Nerdinger, F. W. (2011). Grundlagen der Organisationspsychologie. Basiswissen und Anwendungshinweise. Stuttgart: Schäffer-Poeschel.

Segerer, R., Marx, A., Stanat, P., Schneider, W., Roick, T. \& Marx, P. (2013). Determinanten der Lesekompetenz bei Jugendlichen nicht deutscher Herkunftssprache. Zur Bedeutung der Spracherwerbsreihenfolge im Falle von Mehrsprachigkeit. In N. Jude, E. Klieme (Hrsg.), PISA 2009 - Impulse für die Schul- und Unterrichtsforschung (Beiheft der Zeitschrift für Pädagogik, Bd. 59, S. 111 -131). Weinheim: Beltz.

Statistisches Bundesamt (2016). Schulen auf einen Blick. Zugriff am 22.02.2018 unter https://www.destatis.de/DE/Publikatio nen/Thematisch/BildungForschungKultur/Schulen/Broschuere SchulenBlick0110018169004.pdf?_blob=publicationFile

Steinert, B., Klieme, E., Maag Merki, K., Döbrich, P., Halbheer, U. \& Kunz, A. (2006). Lehrerkooperation in der Schule: Konzeption, Erfassung, Ergebnisse. Zeitschrift für Pädagogik, 52, 185 - 203.

Teerling, A. \& Köller, O. (2019). Implementationsprozesse in Schulen. Herausforderungen und Perspektiven. Psychologie in Erziehung und Unterricht, 66, 3-5. https://doi.org/10.2378/ peu2019.art02d

Tarelli, I., Valtin, R., Bos, W., Bremerich-Vos, A. \& Schwippert, K. (2012). IGLU 2011: Wichtige Ergebnisse im Überblick. In W. Bos, I. Tarelli, A. Bremerich-Vos \& K. Schwippert (Hrsg.), IGLU 2011. Lesekompetenzen von Grundschulkindern in Deutschland im internationalen Vergleich (S. 11-26). Münster: Waxmann.

Warm, T. A. (1989). Weighted likelihood estimation of ability in item response theory. Psychometrika, 54, 427-450.

Weishart, H. (2008). Innovationen in Unternehmen und Schulen. Faktoren und Strategien. Berlin: Wissenschaftlicher Verlag Berlin. 
Zacher, H., Felfe, J. \& Glander, G. (2008). Lernen im Team: Zusammenhänge zwischen Personen- und Teammerkmalen und der Leistung von Multiplikatoren. Zeitschrift für Arbeits- und Organisationspsychologie, 52, 81 -90. https://doi.org/10.1026/ 0932-4089.52.2.81

Zentrale Koordinierungsstelle „SINUS an Grundschulen“ (2018). SINUS an Grundschulen. Skalendokumentation. Kiel.

Zitzmann, S. (2018). A computationally more efficient and more accurate stepwise approach for correcting for sampling error and measurement error. Multivariate Behavioral Research, 53, 612 - 632. https://doi.org/10.1080/00273171.2018.1469086

\section{Historie}

Eingegangen: 04.10.2018

Revision eingegangen: 30.11.2019

Onlineveröffentlichung: 07.04.2020

\section{Förderung}

Das Teilprojekt „Evaluation von Konzepten und Maßnahmen der fachübergreifenden Leseförderung im Primarbereich“" (BiSS-EvalLesen) wird im Rahmen des Programms „Bildung durch Sprache und Schrift" (BiSS) vom Bundesministerium für Bildung und Forschung (BMBF) gefördert (Förderkennzeichen: 01JI1504 A\&01JI1504B).

\section{Autorenschaften}

Ein verwandter Artikel der Autorinnen und Autoren basierend auf dem gleichen Datensatz (Schulleitungen und Lehrkräfte) ist bereits in Psychologie in Erziehung und Unterricht erschienen (2018).
Annika Teerling, M. Ed., M. A.

Prof. Dr. Olaf Köller

Leibniz-Institut für die Pädagogik

der Naturwissenschaften und Mathematik (IPN)

Universität Kiel

Olshausenstraße 62

24106 Kiel

teerling@leibniz-ipn.de

\section{Dr. Steffen Zitzmann}

Institut für pädagogisch-psychologische

Lehr- und Lernforschung (IPL)

Christian-Albrechts-Universität zu Kiel

Olshausenstraße 75

24118 Kiel

\section{Jennifer Igler, M. A.}

Theresa Schlitter, M. A.

Dr. Annika Ohle-Peters

Prof. Dr. Nele McElvany

Institut für Schulentwicklungsforschung (IFS)

Technische Universität Dortmund

Vogelpothsweg 78

44227 Dortmund 